\title{
El Inversor: un ejercicio gamificado para mercados de valores
}
The Investor: a stock market gamified experience

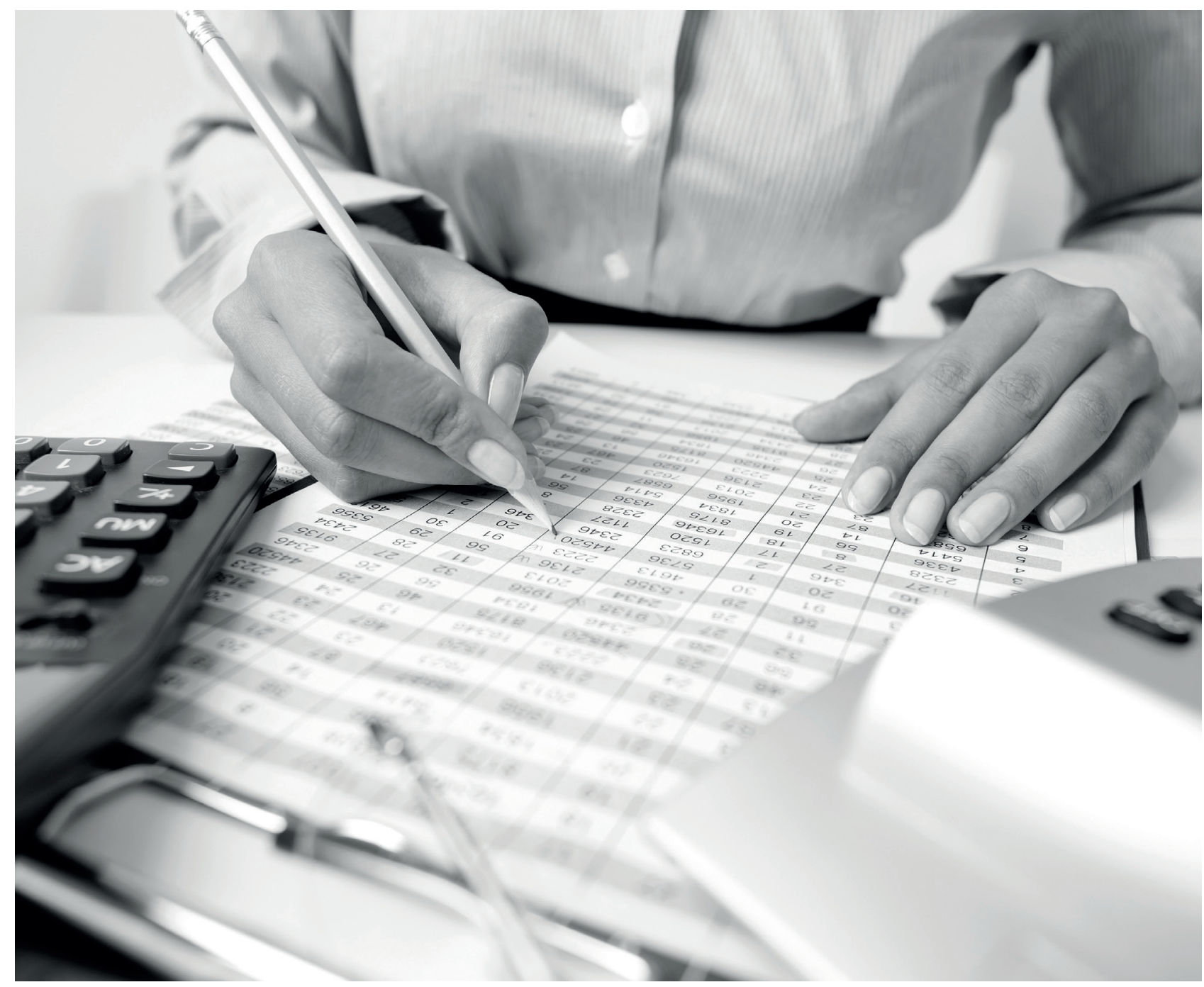

Fuente: Freepik.com Licencia Creative Commons 


\title{
El Inversor: un ejercicio gamificado para mercados de valores ${ }^{1}$
}

\author{
The Investor: a stock market gamified experience
}

\author{
Diego Felipe Lobo-Rueda², Miguel Ángel Lobo-Rueda ${ }^{3}$
}

Artículo recibido en abril 27 de 2020; artículo aceptado en agosto 03 de 2020

\begin{abstract}
Este artículo puede compartirse bajo la Licencia Creative Commons Atribución-NoComercial-Compartirlgual 4.0 Internacional y se referencia usando el siguiente formato: Lobo-Rueda, D. F. y Lobo-Rueda, M. A. (2021). El Inversor: un ejercicio gamificado para mercados de valores. I+D Revista de Investigaciones, 16(1), 75-86. http://dx.doi.org/10.33304/revinv.v16n1-2021007.
\end{abstract}

\begin{abstract}
Resumen
En Colombia se presenta un desconocimiento en materia de educación financiera, lo cual contrasta con un aumento del interés por aprender temas enfocados en finanzas. Esto, en términos académicos, refleja una brecha entre la existencia de herramientas de enseñanza a niveles de educación media y superior, y su efectividad real en los estudiantes. A fin de contribuir a llenar esa brecha y contribuir a la alfabetización financiera a nivel universitario, se propone el diseño de un ejercicio lúdico dinámico y amigable relacionado con una simulación de inversión en la bolsa de valores de una empresa específica. Para el diseño, se utilizó la metodología de design thinking. Resultó una lúdica que busca proporcionar una introducción para los estudiantes al tema de los mercados de capitales y generar un interés orgánico en ellos. A partir de esto, se espera probar la lúdica en un grupo piloto y validar su funcionamiento, a fin de aportar, por medio de la gamificación, bases sólidas que den paso al progreso de la alfabetización financiera en la educación superior.
\end{abstract}

Palabras clave: Gamificación, decisión, dinero, mercado de valores.

\begin{abstract}
In Colombia, there is a lack of knowledge in financial education, which contrasts with a growing interest in learning about topics focused on finance. In academic terms, this reflects a gap between the existence of teaching tools at the middle and higher education levels and its real effectiveness for students. In order to help fill that gap, one proposal focuses on contributing to financial literacy at the university level. Thus, this article describes the design of a dynamic and friendly recreational exercise related to a simulation of investment in the stock market of a specific company. The purpose is to provide an introduction for students to the subject of capital markets and to generate organic interest in them. From this design, it is expected to test the game in a pilot group and validate its operation, thus providing gamification, solid foundations that give way to the progress of financial literacy in higher education.
\end{abstract}

Keywords: Gamification, decision, money, stock market.

\footnotetext{
${ }^{1}$ Artículo de investigación de enfoque cualitativo, perteneciente al área de ciencias sociales, subárea de educación financiera, desarrollado en el Laboratorio GALEA, adscrito al Grupo de Investigación Finance \& Management, fue financiado por la Universidad Industrial de Santander (Bucaramanga, Colombia). Dirección: carrera 27 calle 9, PBX: (57) (7) 6344000. Fecha de inicio: 12.4.2020. Fecha de terminación: 11.06.2020. ${ }^{2}$ Estudiante de Ingeniería Industrial de la Universidad Industrial de Santander. Vinculado al Laboratorio GALEA adscrito al Grupo de Investigación Finance \& Management de la Universidad Industrial de Santander (Bucaramanga, Colombia). Dirección: carrera 27 calle 9, PBX: (57) (7) 6344000. ORCID ID: https://orcid.org/0000-0001-5034-2592. Correo electrónico institucional: diego2201157@correo.uis.edu.co.

${ }^{3}$ Estudiante de Ingeniería Industrial de la Universidad Industrial de Santander, Vinculado al Laboratorio GALEA adscrito al Grupo de Investigación Finance \& Management de la Universidad Industrial de Santander (Bucaramanga, Colombia). Dirección: carrera 27 calle 9, PBX: (57) (7) 6344000. ORCID ID: https://orcid.org/0000-0002-6618-9024. Correo electrónico institucional: miguel. lobo@correo.uis.edu.co.
} 
Diego Felipe Lobo-Rueda, Miguel Ángel Lobo-Rueda El Inversor: un ejercicio gamificado para mercados de valores

\section{Introducción}

El desempeño de una asignatura de pregrado, como es el caso de Mercado de capitales, depende de varios aspectos. Entre estos, la motivación por parte del estudiante o las bases de conocimiento anteriormente adquiridas en áreas fundamentales de la formación, como la educación media o bachillerato. La motivación puede dar diferencias significativas al desempeño académico de los estudiantes. La falta de esta representa desapego por el proceso de aprendizaje o un sentimiento de despropósito por el proceso de formación (TorresBarreto, 2018). Adicionalmente, el fenómeno puede ser explicado por un deficiente conocimiento en educación financiera desde la educación media. Esto lo demuestra Asobancaria en una encuesta realizada en 2018 a diferentes centros educativos del país. Los resultados demuestran que los estudiantes poseen un conocimiento insuficiente en el área de educación financiera (Castro et al., 2018). Dada esta deficiencia de conocimiento en el área financiera, se propone el diseño de una lúdica titulada "El Inversor", la cual busca dar un apoyo gamificado a ambas posibilidades e introducir al estudiante en la materia de Mercado de capitales.

El ejercicio lúdico que se describe en este manuscrito comprende las áreas de finanzas y análisis de inversiones, que, impartidas conjuntamente, tienen el propósito de proporcionar a los estudiantes una formación sistemática y una comprensión de los fundamentos de estas dos áreas y de la aplicación crítica de los métodos de análisis (Pontifícia Universidade Católica do Rio de Janeiro, 2016).

El propósito de El Inversor es ayudar a los estudiantes a potenciar sus conocimientos en mercados financieros, al tiempo que refuerza sus competencias analíticas acerca del mercado de capitales, y hace de esta su principal diferencia con las lúdicas ya existentes. El Inversor pretende dar una introducción básica al mercado financiero, así como proporcionar al estudiante un primer contacto con los temas de mercados de capitales e inversiones en la bolsa de valores, aplicando de forma didáctica aspectos de la carrera de ingeniería industrial.

\section{Gamificación y aprendizaje}

La gamificación es la aplicación de principios y elementos propios del juego en contextos ajenos al juego (Deterding et al., 2011). Tiene por objetivo atraer la atención de los usuarios y motivarlos a ejecutar determinadas acciones (Zichermann y Cunningham, 2011). Hay que tener en cuenta que el objetivo de la gamificación no se centra en el entretenimiento, sino que debe tener una clara noción de sus objetivos dentro de sus interacciones. Al no aplicarse de forma asertiva, resulta en un despropósito dentro del marco educativo que abarca la gamificación. Esto puede suceder al no poseer unos objetivos claros o tener una narrativa deficiente que delimitan la motivación del estudiante. Sin embargo, una aplicación asertiva desemboca en el desarrollo de habilidades como la resolución de conflictos por medio del mejoramiento del área comunicativa y estratégica, y conocimientos particulares que se buscan generar en los alumnos por medio de la lúdica (Peris, 2015). De la misma manera, los profesores universitarios buscan incluir esta estrategia de aprendizaje en sus clases, con el fin de potenciar estas habilidades anteriormente mencionadas en sus alumnos por medio de actividades. Esto supone un reto a la hora de motivar a los estudiantes y generar un sentimiento de compromiso en la asignatura (Contreras y Eguia, 2016).

La gamificación se puede dar de dos maneras: "gamificación de capa fina" y "gamificación profunda". Su diferencia no radica en un objetivo común de aprendizaje, sino en el estilo de interacción con los estudiantes. La gamificación de capa fina basa su entretenimiento en premios y jerarquización de sus estudiantes en el desarrollo de la actividad, mientras que la gamificación profunda capta la atención de los estudiantes por medio de una motivación intrínseca; es decir, involucra a los estudiantes por medio de un sentimiento de gusto por sus acciones dentro de la actividad (Prieto et al., 2014).

El uso delagamificación como herramienta de aprendizaje debe tener una serie de objetivos o enfoques ante su fin académico. Tales objetivos se resumen en hacer un juego/actividad entretenida, autentica, reglamentada y fundamentalmente motivante y placentera. La razón de existir de estos objetivos es sumergir a los alumnos en una realidad única e inclusiva, en la cual las reglas representan la rigurosidad del aprendizaje e indirectamente estimulan la creatividad e innovación de los estudiantes por alcanzar el éxito o resolución de un conflicto (Luis-Pascual, 2015).

En este contexto, el desarrollo de la gamificación, también llamada ludificación, puede verse como un ejemplo de la continua renovación de la práctica formativa. Se trata de una de las tendencias de innovación educativa que se puede usar para soportar el proceso de enseñanza y aprendizaje. Ayuda tanto a los maestros, en el plano de mejorar el proceso de seguimiento, como a los estudiantes, en el de aumentar su motivación para aprender de nuevas maneras y disfrutar sus actividades académicas (Acosta-Medina et al., 2020).

\section{Importancia de la educación financiera}

La cultura financiera ha sido abordada por numerosos autores, como Polania et al. (2016), quienes remarcan la importancia que esta toma en la vida personal y en la economía nacional. Adicionalmente, es relevante hacer énfasis en la importancia de reforzar la educación financiera por medio de métodos innovadores, como la gamificación. Alineado a esto, Acosta-Medina et al. (2020) exponen, por medio de mapeos de tendencias y evaluación de distintos patrones, los resultados favorables del uso de las herramientas gamificadas. 
Son algunos de estos efectos el mejoramiento en el compromiso, motivación y rendimiento.

El término cultura o educación financiera se refiere a "la curiosidad por el entendimiento y conocimiento de las llamadas finanzas personales, incluyendo los conceptos de ingresos y gastos, y fundamentalmente, el del ahorro" (Hernández, 2015).

De acuerdo con la definición aportada por la Organización para la Cooperación y el Desarrollo Económicos (OCDE), la educación financiera es definida como:

El proceso por el que los inversores y consumidores financieros mejoran su comprensión de los productos financieros, conceptos y riesgos, $y$, a través de la información, la enseñanza y/o el asesoramiento objetivo, desarrollan las habilidades y confianza precisas para adquirir mayor conciencia de los riesgos y oportunidades financieras, tomar decisiones informadas, saber dónde acudir para pedir ayuda y tomar cualquier acción eficaz para mejorar su bienestar financiero. (OECD, 2005)

De estas definiciones surgen preguntas como ¿dónde se sitúa el nivel de cultura financiera en los colombianos? o ¿qué se está haciendo para aumentar la actividad financiera? Una primera aproximación la da Domínguez (2013), quien indica que, dada la importancia de la educación, esta debe ser impartida a los ciudadanos de cualquier edad, ya que se trata de un proceso continuo a lo largo de toda la vida, y se recomienda empezar desde una temprana edad.

Otros autores afirman que existe cierta ignorancia generalizada en la población respecto a conceptos financieros básicos, como inflación, tasa de interés, relación entre riesgo y rentabilidad, y sobre el funcionamiento del mercado de capitales (Hernández, 2015; Plata-Gómez, y Caballero-Márquez, 2020).

\section{El papel de la gamificación en la educación financiera}

Ante esta problemática, se evidencia un nivel de educación financiera insuficiente en gran parte de la población colombiana, a pesar de que existen programas nacionales destinados a impulsar dicha alfabetización financiera. En una investigación desarrollada por García et al. (2013), se aborda la educación financiera en América Latina y el Caribe. A partir de encuestas realizadas en los ámbitos regional y nacional en varios países de América Latina y el Caribe, se realiza un informe de la situación actual y perspectiva, que indica que cuanto más aumenta la educación financiera y/o inclusión en esta área en la población, los índices de pobreza tienden a disminuir en proporción. Además, la disposición por parte de entes administrativos, como bancos, se traduce en una combinación entre acceso a productos y servicios, junto con un programa de sensibilización y educación financiera. Por lo tanto, resulta altamente promisoria la ejecución de proyectos con fines de alfabetización financiera en la población (García et al., 2013).

Teniendo en cuenta lo expuesto anteriormente, desde el Laboratorio de innovación educativa GALEA, y teniendo como referente el artículo de investigación "Análisis descriptivo de experiencias gamificadas para enseñanza y aprendizaje en educación superior en ingeniería" (Lobo-Rueda et al., 2020), se propone la adecuación de conceptos propios de la educación financiera para su difusión didáctica dentro del currículum académico del programa de Ingeniería Industrial, con perspectiva a su aplicación en otros escenarios de la comunidad estudiantil. La lúdica El Inversor orienta su desarrollo en los conceptos fundamentales de la gamificación, y aprovecha así varias de las ventajas que brinda, como el aumento de la motivación y el fomento de la creatividad. De esta forma, se asegura un acercamiento amigable a la serie de conceptos referentes a educación financiera que se introducirán, y, a partir del componente lúdico (la simulación del comportamiento de la bolsa de valores), se pretende de forma simultánea ver dichos conceptos y su importancia. De esta forma se ofrece formación teórica y práctica en un ambiente pedagógico.

\section{Incorporación de la educación financiera en la ingeniería}

En Colombia se hace evidente una ausencia de educación financiera en las escuelas, y así mismo en la educación superior. Esta situación no es ajena a otros países. En Estados Unidos y en Europa se evidenció un desarrollo exponencial a partir del año 2008. Esta fecha dio lugar a una significante crisis financiera generada por la carencia de educación en el área (Villada et al., 2017), ya que finalmente fueron las personas naturales quienes tomaron la decisión de solicitar deudas por encima de sus posibilidades, adquirir una vivienda inflada de precio o invertir en títulos basura por desconocimiento de la calidad de la inversión (Feldkircher, 2014). Por tanto, impartir una cultura financiera es de gran relevancia, dado que permitirá no solo mejorar la calidad de vida, sino también proteger la economía nacional de crisis como la referente al colapso de la burbuja inmobiliaria, al contar con una población con mayor conciencia sobre sus decisiones financieras.

El estudiante universitario actual será el profesional del futuro. Por esto es necesario inculcar una cultura de ahorro e inversión que le brinde las herramientas necesarias para poder acumular capital y desenvolverse en un ambiente laboral donde pueda gozar de estabilidad económica.

Existen ejemplos como Escocia, donde desde 2008 la educación financiera es obligatoria en el currículum de las escuelas. 
Diego Felipe Lobo-Rueda, Miguel Ángel Lobo-Rueda El Inversor: un ejercicio gamificado para mercados de valores

En la Tabla 1 se expone el efecto que tuvo la incorporación de proyectos de aula sobre la valoración de un curso de aprendizaje sobre mercados y bolsa de energía, que constituyó una mejoría notable en la percepción de los estudiantes en cuanto a la capacidad de despertar interés, el empleo de recursos didácticos y el apoyo a las actividades de aprendizaje independientes (Villada et al., 2017).

Tabla 1

Evaluación de cursos antes y después de implementar una metodología de aprendizaje por proyectos

\begin{tabular}{|c|c|c|}
\hline Evaluación del curso & Antes & Después \\
\hline $\begin{array}{l}\text { La metodología del curso tiene la capacidad } \\
\text { de despertar el interés de los estudiantes }\end{array}$ & 4,14 & 4,59 \\
\hline Se emplean recursos didácticos adecuados & 4,15 & 4,62 \\
\hline $\begin{array}{l}\text { El profesor es eficiente en el uso del tiempo } \\
\text { de clase y actividades }\end{array}$ & 4,45 & 4,65 \\
\hline $\begin{array}{l}\text { Existe orden, coherencia y claridad en la } \\
\text { exposición de los temas }\end{array}$ & 4,23 & 4,59 \\
\hline $\begin{array}{l}\text { El profesor es puntual y asiste a todas las } \\
\text { sesiones de clase }\end{array}$ & 4,66 & 4,61 \\
\hline $\begin{array}{l}\text { Se apoyan las actividades de aprendizaje } \\
\text { independientes }\end{array}$ & 4,20 & 4,60 \\
\hline $\begin{array}{l}\text { Es importante el curso dentro de su plan de } \\
\text { estudios }\end{array}$ & 4,62 & 4,69 \\
\hline $\begin{array}{l}\text { El curso es interesante y actualizado en sus } \\
\text { contenidos }\end{array}$ & 4,48 & 4,74 \\
\hline $\begin{array}{l}\text { Considera usted que se lograron los } \\
\text { objetivos formulados en el curso o actividad } \\
\text { curricular }\end{array}$ & 4,41 & 4,66 \\
\hline
\end{tabular}

Fuente: Adaptado de Villada et al. (2017).

La Tabla 1 corresponde a los resultados de incorporación de estrategias metodológicas orientadas a la educación financiera (en este caso, la metodología que se implementó fue un aprendizaje por proyectos), que repercute tanto en una mayor formación del estudiante como en una mayor disposición de este frente a los componentes teóricos impartidos. Esto facilita la dinámica de la clase y el compromiso del estudiante por aprender y reconocer la importancia que la educación financiera supone para su vida diaria y profesional.

\section{Metodología}

El diseño y desarrollo de la lúdica El Inversor fue concebido como un proyecto unificado, del cual surgió el presente estudio de carácter descriptivo. La realización de los procesos de diseño, desarrollo y validación se basó en la metodología de design thinking, que, según Leichter (2011), "promueve una cultura para la creación de prototipos con una clara inclinación por la acción". La metodología usada se vio reflejada en 5 etapas: empatizar, definir, idear, prototipar y validar.

En un primer momento, se realizó una investigación sobre el público objetivo y las iniciativas de igual o similar naturaleza, con lo que se abarcó la etapa de empatizar. En segundo lugar, al llegar a la etapa de definir, se determinaron las condiciones específicas y necesidades puntuales del contexto educativo a impactar. Seguido de esto, se realizó un brainstorming para definir la dinámica y la mecánica a utilizar para solventar las necesidades detectadas. Posteriormente, se creó una lúdica para el aprendizaje de la educación financiera, enfocada en el mercado de valores. Finalmente, se realizó un ejercicio de validación con 10 estudiantes de ingeniería industrial pertenecientes a una universidad de Santander. En el proceso se utilizó como instrumento la observación, y al finalizar el ejercicio se contó con un espacio de retroalimentación entre los participantes, lo que permitió identificar los aspectos a mejorar en la lúdica.

La lúdica busca fomentar el desarrollo de habilidades de toma de decisiones y una introducción a conceptos básicos referentes a la educación financiera.

\section{Resultados}

\section{Fijar el objetivo formativo de la lúdica}

Por medio del método de gamificación, en conjunto con la importancia de la educación financiera y el énfasis en el bajo desempeño de inversión en la bolsa de valores. El Inversor busca, de forma lúdica, brindar una introducción al tema de mercado de capitales con énfasis en la inversión en la bolsa de valores. Con la actividad no se pretende crear expertos en el tema, sino dar un primer contacto por medio del aprendizaje o afianzamiento de conceptos básicos con respecto a mercado de capitales, ya que funciona como una simulación interactiva con los estudiantes, quienes podrán aprender, con base en experiencias de juego, y así obtendrán conocimientos que pueden resultar útiles en su vida académica $y / 0$ laboral.

\section{Habilidades para desarrollar}

La lúdica El Inversor busca desarrollar principalmente las habilidades de toma de decisiones.

\section{Materiales para el desarrollo de la lúdica}

Con el fin de desarrollar correctamente la lúdica se utilizarán los siguientes materiales:

- Pósits de distintos colores para compra/venta por cada equipo (ver Figura 1).

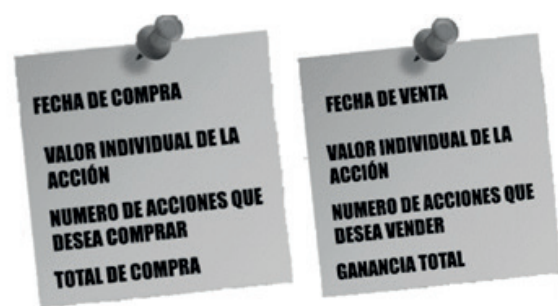

Figura 1. Formato pósit compra/venta. Fuente: Autores. 
- 2 lapiceros por cada equipo de juego.

- Legos suficientes, los cuales van a representar visualmente el número de acciones que el equipo de inversionistas posea; esto con el fin de tener una perspectiva cuantitativa de las acciones.

- 8 preguntas de educación financiera de nivel introductorio en el tópico.

- Banderines de diferentes colores; se entregará uno a cada equipo.

- Una tabla con los equipos correspondientes y momentos de compra y venta suficientes para el desarrollo de la lúdica (véase en el Anexo 1).

- 8 noticias hipotéticas acordes al comportamiento del historial de las acciones conforme se vaya desarrollando la actividad. Las noticias deben contener 3 datos: 1) el número de la noticia, que corresponde al orden cronológico en el cual se encuentra con respecto a las demás; 2) El encabezado de la noticia que resume la situación que afecta positiva o negativamente el comportamiento de las acciones en la bolsa de valores, esto con el fin de que el estudiante infiera esta información y la use a su beneficio, y 3) Una fecha específica, que el estudiante debe tener en cuenta para saber cuándo realizar o no una inversión (ver Figura 2).

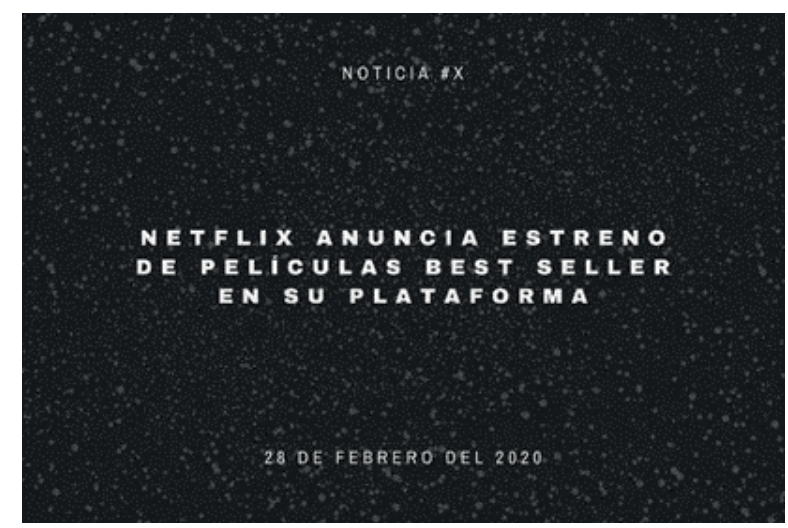

Figura 2. Ejemplo tarjeta noticia. Fuente: Autores.

- Una ruleta virtual dividida en 12 partes. En esta se encontrará una posibilidad de obtener directamente una noticia, una de perder el turno, seis de realizar un reto con sus compañeros y cuatro de recibir una pregunta acorde al nivel de educación financiera de los participantes (ver Figura 3).

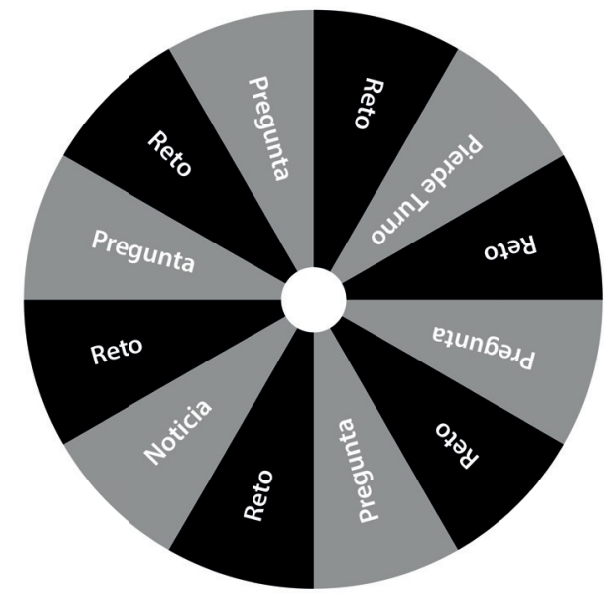

Figura 3. Ruleta de decisiones. Fuente: Autores.

- Una cuenta en la herramienta TradingView, que se puede crear desde el enlace: https://www. tradingview.com/.

- Un computador con acceso a internet y un proyector de video.

\section{Desarrollo de la lúdica}

Una vez se cuenta con los materiales expuestos previamente, el desarrollo se hará de la siguiente manera:

I. Lo primero que se debe hacer es conformar equipos de 3 personas. La actividad se debe realizar con un mínimo de 3 equipos y un máximo de 6 . Cada grupo deberá identificarse con una letra del alfabeto. Se asignarán como Equipo A, Equipo B y así sucesivamente.

II. A cada equipo participante se le debe entregar un banderín con un color distintivo, esto con el fin de facilitar el reconocimiento de prioridad en cuanto al desarrollo de cada actividad de simulación en la bolsa, además de dos lapiceros y dos paquetes de 30 pósits (cada paquete de un color diferente).

III. A partir de este momento comienza la lúdica con los estudiantes. Se utilizará una ruleta virtual que va a estar dividida en 12 partes iguales. La información correspondiente a la composición de la ruleta se encuentra en el apartado de materiales para el desarrollo de la lúdica (ver Figura 3). A continuación, cada equipo deberá escoger un integrante con el fin de girar la ruleta. De esta manara los participantes probarán suerte en esta actividad. Se procederá de la siguiente manera según las diferentes opciones:

- Si el estudiante resulta en la opción de noticia, recibirá una noticia aleatoria, sin la necesidad de pasar por algún reto o pregunta. 
- Si el estudiante resulta en la opción de pierde turno, cederá el paso al siguiente participante según el orden establecido, y no recibirá ni una noticia ni penalización extra.

- Si el estudiante resulta en la opción de reto, deberá cumplir un reto de los descritos en el Anexo 2. Si se cumple con satisfacción dicho reto, recibirá una noticia como premiación; sin embargo, si no cumple con lo establecido, no recibirá premiación y se continuará con la actividad lúdica.

- Si el estudiante resulta en la opción de pregunta, deberá responder a una pregunta seleccionada por el colaborador o auxiliar a cargo. Si se responde asertivamente, dicha pregunta recibirá como premiación una noticia; en caso contrario, no recibirá premiación y se continuará con la actividad lúdica.

En este punto se dará por terminada la repartición de noticias. Es decir, no se contará con más información hasta el final de la actividad.

IV. En esta parte de la lúdica se utilizarán los banderines, los pósits, las noticias, los legos y la aplicación TradingView para desarrollar la simulación de inversión en la bolsa de valores. La herramienta TradingView permite ver el comportamiento del valor nominal de una acción de una empresa específica; además, permite ver las fechas en las cuales se efectúan los cambios del valor en la acción, y las barras representan el alza o la baja en el precio del título de valor (ver Figura 4).

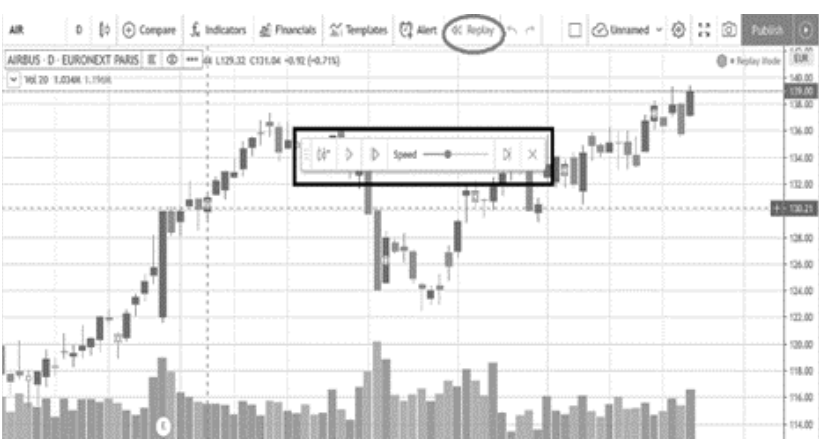

Figura 4. TradingView: Control del histórico. https://es.tradingview. com/.

Para el uso de esta aplicación, es fundamental conocer el funcionamiento de ciertas herramientas dentro de esta. La primera se encontrará en la parte superior de la aplicación en una barra de tareas, denominada con el nombre de Replay, que será utilizada para la reproducción del historial de la empresa; al seleccionarlo se desplegará una barra con diferentes opciones (ver Figura 5)

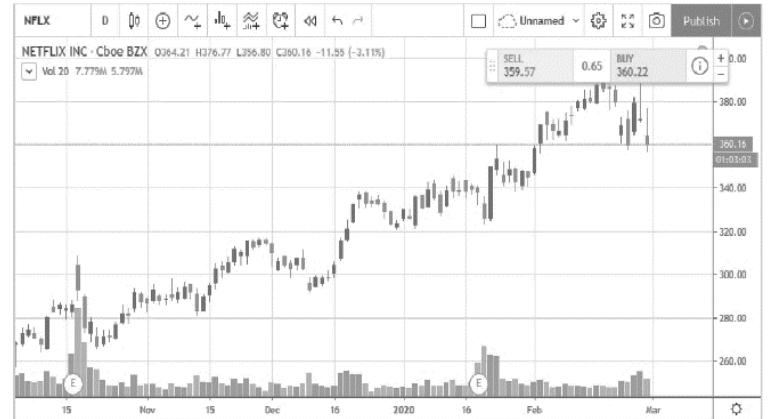

Figura 5. TradingView, Histórico de la empresa a invertir. https:// es.tradingview.com/.

En la barra de opciones del Replay, el primer botón se utiliza para volver a un momento específico del histórico de la acción. Para esto debemos primero seleccionar esta opción, y segundo seleccionar el momento. La siguiente opción para tener en cuenta es la velocidad de reproducción del historial de la empresa. Esta deberá ser configurada en Speed; sin embargo, se recomienda una velocidad de reproducción de 1 segundo (ver Figura 6).

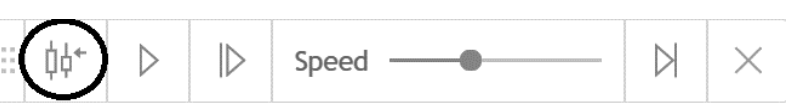

Figura 6. Control de velocidad. https://es.tradingview.com/.

Al tener conocimiento del uso de esta herramienta dentro de la plataforma, solo resta conocer el comportamiento de la acción nominal de una empresa específica, con el fin de usar esta interpretación en el ejercicio gamificado. En la aplicación de esta fase se debe proyectar el histórico para los equipos y comenzar la reproducción del historial una vez se haya explicado la dinámica de la actividad lúdica. A continuación, se dará una explicación breve del funcionamiento de cada elemento en esta fase:

- Los banderines funcionarán como indicativo de interrupción en el historial. Al momento que un equipo levante el banderín, debe detenerse de inmediato la reproducción. Así serán turnos únicos; es decir, solo da cabida a una interacción con la bolsa por equipo en cada turno.

- Los legos son la representación visual de las acciones en el mercado. Al momento de comprar una acción cada unidad de lego funciona como la representación de esta compra; así mismo, al momento de vender acciones se deberán devolver el número de legos proporcional al número de acciones vendidas.

- Los pósits y la tabla funcionan como el historial según las decisiones de cada equipo con respecto al comportamiento previo de la empresa. En el momento que un equipo tome la decisión de comprar o vender una acción, estos deberán dar una información específica en un pósit dependiendo de esta decisión.

- Si la decisión es comprar una acción, la información 
correspondiente es: (1) fecha de compra, (2) valor individual de la acción, (3) número de acciones que desea comprar y (4) precio total de compra.

- Si la decisión es vender una acción, la información correspondiente es: (1) fecha de venta, (2) valor individual de la acción, (3) número de acciones que desea vender y (4) ganancias o pérdidas por la venta.

Enelmomento que los participantes tengan conocimiento del funcionamiento de cada parte de esta fase, se da inicio a la reproducción del historial, y los estudiantes pueden comenzar su interacción con la actividad. Cuando haya concluido la reproducción de dicho historial, se dará por terminado el ejercicio gamificado.

V. Al finalizar la reproducción del historial, se decide el equipo ganador que corresponde a quienes hayan generado mayor cantidad de ingresos con sus inversiones.

Como paso final, se realizó un ejercicio de validación, en el que se simuló una interacción en la bolsa de valores. En el ejercicio participaron 10 estudiantes de ingeniería industrial pertenecientes a una universidad de Santander. Para el desarrollo, se utilizaron los materiales propios de la lúdica y se siguieron los pasos anteriormente descritos. En un principio se identificó la necesidad de distinguir los equipos con banderines de un color característico de cada uno. Esto debido a la dificultad que se observó al no poder distinguir de forma eficaz a los participantes. Adicionalmente, fue fundamental la aclaración de aspectos clave de la herramienta de TradingView, con el fin de agilizar el desarrollo de la experiencia gamificada. Algunos aspectos se relacionan con cómo se representan el alza y la baja de precios en la simulación, cómo estas pueden llegar a fluctuar con el paso del tiempo y cómo se ven en relación con las noticias repartidas en la primera fase de la actividad.

Durante el ejercicio de validación, los autores observaron en detalle el comportamiento de los participantes en la lúdica, su característica, la claridad de los pasos y las actividades a desarrollar. Así mismo, se contó con un espacio de retroalimentación, conclusiones y percepciones al finalizar la lúdica. Esto permitió identificar la necesidad de representar de forma tangible las acciones que los estudiantes compran o venden en la herramienta. Por tanto, se añadió la idea de utilizar legos como esta representación física en la lúdica. Se observaron errores y aspectos por mejorar, con lo que se corrigieron concepciones iniciales y se mejoró el componente contextual de las dinámicas de juego.

\section{Discusión y conclusiones}

Desde la perspectiva educativa, la lúdica El inversor da un precedente para el abordaje de esta temática. Aprovechando la tendencia de la gamificación, este ejercicio, al ser comparado con estrategias más tradicionales, muestra un abordaje más orgánico y desinhibido en temáticas de difícil interiorización y difusión académica, como el comportamiento de los títulos de valor. Esta propuesta es particular, ya que implica un adelanto en el contenido necesario para el mejoramiento de los estándares en educación financiera en América Latina, y la utilización de estrategias de vanguardia para el aumento del engagement en el alumnado.

Para realizar este ejercicio de gamificación, se hizo necesario el uso de una herramienta virtual que permitiese ver el histórico de la empresa a invertir, la cual es el elemento principal de la lúdica. Se escogió una empresa con fluctuaciones relevantes de dinero a lo largo del histórico, con el fin de que los inversionistas puedan percibir la volatilidad del mercado, y a partir de esta se escogieron momentos clave, fechas en donde sucedieron las fluctuaciones, para así elaborar tarjetas de noticias con las cuales los inversores podrían inferir sobre el comportamiento del mercado en dicha fecha. Las tarjetas de noticias son un recurso limitado, y para distribuirlas se realizarán varias rondas de sorteo, haciendo uso de una ruleta que cuenta con retos y preguntas pertinentes a los temas que aborda la lúdica.

Este ejercicio lúdico es un caso de estudio que evidencia que la utilización de la metodología design thinking tiene una amplia eficacia en la creación de herramientas para el sector educativo.

El objetivo de la lúdica El Inversor está en línea con el objetivo académico y la metodología interactiva e innovadora para el aprendizaje del Laboratorio de innovación educativa GALEA. Se trata de una lúdica que busca contribuir y complementar los contenidos de materias usuales dentro del pénsum de programas en ingeniería industrial, como es el caso de Mercado de capitales. Por medio de la lúdica se espera generar mayor interés en los estudiantes de ingeniería industrial por adquirir un primer contacto introductorio a este tema. En pocas palabras, la lúdica posibilita la construcción de conocimiento para la independencia financiera. Esto significa un aumento en la calidad de vida, debido al mantenimiento sólido de un estado del bienestar, lo que crea riqueza y prosperidad.

Con la lúdica se busca que los estudiantes perciban información básica y esencial en el área de mercado de capitales y educación financiera, por medio de sus interacciones en la actividad. Por lo tanto, fue creada con la finalidad de mantener al estudiante participando el mayor tiempo posible dentro del desarrollo de la actividad, mediante las herramientas de juego de forma entretenida y sin perder el sentido de sus acciones. 
Diego Felipe Lobo-Rueda, Miguel Ángel Lobo-Rueda El Inversor: un ejercicio gamificado para mercados de valores

\section{Agradecimientos}

Agradecemos a la profesora Martha Liliana Torres Barreto, directora del Grupo de Investigación Finance \& Management y directora del Laboratorio GALEA de la Universidad Industrial de Santander, por su apoyo en el diseño de los casos, así como por su supervisión y comentarios en la construcción de este manuscrito. También queremos manifestar un agradecimiento a Brayan Esneider Monroy Chaparro, por sus aportaciones a la construcción del marco teórico y el ejercicio gamificado de El Inversor; y finalmente a la Universidad Industrial de Santander, por prestar las instalaciones y el material para las pruebas piloto y el refinamiento de la lúdica El Inversor.

\section{Referencias}

Acosta-Medina, J. K., Torres-Barreto, M. L. y AlvarezMelgarejo, M. (2020). Literature Mapping About Gamification in the Teaching and Learning Processes. Revista ESPACIOS, 41(11), 26. https://www. revistaespacios.com/a20v41n11/20411126.html

Acosta-Medina, J. K., Torres-Barreto, M. L., AlvarezMelgarejo, M. y Paba-Medina, M. C. (2020). Gamificación en el ámbito educativo: Un análisis bibliométrico. I+D Revista de Investigaciones, 15(1), 30-39. https://doi.org/10.33304/revinv.v15n12020003

Castro, S., Rojas, A y Mantilla, I. (2018). Índice de educación financiera colegios de Asobancaria. (Edición 1127). Semana económica Asobancaria, 1-9. https://www. asobancaria.com/wp-content/uploads/2018/03/11 27C-05-03-2018.pdf

Contreras, R. S. y Eguia, J. L. (2016). Gamificación en aulas universitarias. Institut de la Comunicació, Universitat Autónoma de Barcelona.

Deterding, S., Khaled, R., Dixon, D. y Nacke, L. E. (2011). Gamification: Toward a Definition. Proceedings of the CHI 2011 Gamifi cation Workshop Proceedings, 7-12. ACM.

Domínguez, J. M. (2013). Educación financiera para jóvenes: Una visión introductoria. Serie documentos de trabajo http://www3.uah.es/iaes/publicaciones/ DT_05_13.pdf

Feldkircher, M. (2014). The Determinants of Vulnerability to the Global Financial Crisis 2008 to 2009: Credit Growth and other Sources of Risk. Journal of International Money and Finance, 43, 19-49.

García, N., Grifoni, A., López, J. y Mejía, D. (2013). La educación financiera en América Latina y el Caribe. Situación actual y perspectivas (J. Cuebelo \& M.
Penfold, Eds.). Banco de Desarrollo de América Latina. Serie Políticas Públicas y Transformación Productiva N.o 12., 1-90. https://www.oecd.org/ daf/fin/financial-education/oecd_caf_financial_ education_latin_americaes.pdf

Hernández, T. (2015). La importancia de la educación financiera y su influencia en los futuros estudiantes de Administración y Dirección de Empresas. Anuario jurídico y económico escurialense, 48, 381-400.

Leichter, F. S. (2011). How Fidelity Uses Design Thinking to Perfect Its Website. Harvard Business Review. https:// hbr.org/2011/05/how-fidelity-used-design-think

Lobo-Rueda, M. A., Paba-Medina, M. C. y Torres-Barreto, M. L. (2020). Análisis descriptivo de experiencias gamificadas para enseñanza y aprendizaje en educación superior en ingeniería. Revista ESPACIOS, 41(16), 21. https://www.revistaespacios.com/ a20v41n16/20411621.html

Luis-Pascual, L. (2015). El juego auténtico y las claves de la gamificación del aprendizaje. Inclusao e aprendizagem: desafíos para a escola em IberoAmérica. Sao Paulo, SP: Cultura Económica. https:// doi.org/10.13140/RG.2.1.4084.3288

OECD. (2005). Improving Financial Literacy: Analysis of Issues and Policies. OECD Publishing. https://doi. org/10.1787/9789264012578-en

Peris, F. J. (2015). Gamificación. Education in the knowledge Society, 16(2), 13-15. https://doi.org/10.14201/ eks20151621315

Plata-Gómez, K. R., \& Caballero-Márquez, J. A. (2020). Influencia de los programas de educación financiera sobre el comportamiento de los jóvenes: una revisión de literatura. I+ D Revista De Investigaciones, 15(2), 18-27.

Polania, F., Suaza, C., Arevalo, N. y Gonzalez, D. (2016). La cultura financiera como nuevo motor para el desarrollo economico latinoamaericano. http://hdl. handle.net/10882/8951

Pontifícia Universidade Católica do Rio de Janeiro. (2016). Finanzas y Análisis de Inversiones. http://www. ind.puc-rio.br/es/pesquisa/financas-e-analise-deinvestimentos/

Prieto, A., Díaz, D., Monserrat, J. y Reyes, E. (2014). Experiencias de aplicación de estrategias de gamificación a entornos de aprendizaje universitario. ReVisión. Revista de Investigación en Docencia Universitaria de la Informática, 7(2), 76-92. 
Diego Felipe Lobo-Rueda, Miguel Ángel Lobo-Rueda

El Inversor: un ejercicio gamificado para mercados de valores

Torres-Barreto, M. L. (2018). Herramienta didáctica motivacional basada en gamificación y apoyada en TIC para adquiriry aplicar competencias transversales en estudiantes de ingeniería: MOTIVATIC (N. ${ }^{\circ}$ hal02166319). https://hal.archives-ouvertes.fr/hal-02 16 6319/

Villada, F., López-Lezama, M. J. y Muñoz-Galeano, N. (2017). El papel de la educación financiera en la formación de profesionales de la ingeniería. Formación Universitaria, 10(2), 13-22.

Zichermann, G. y Cunningham, C. (2011). Gamification by

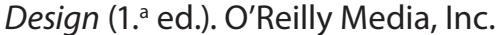




\section{Anexo 1}

Tabla de seguimiento de compra y venta de acciones

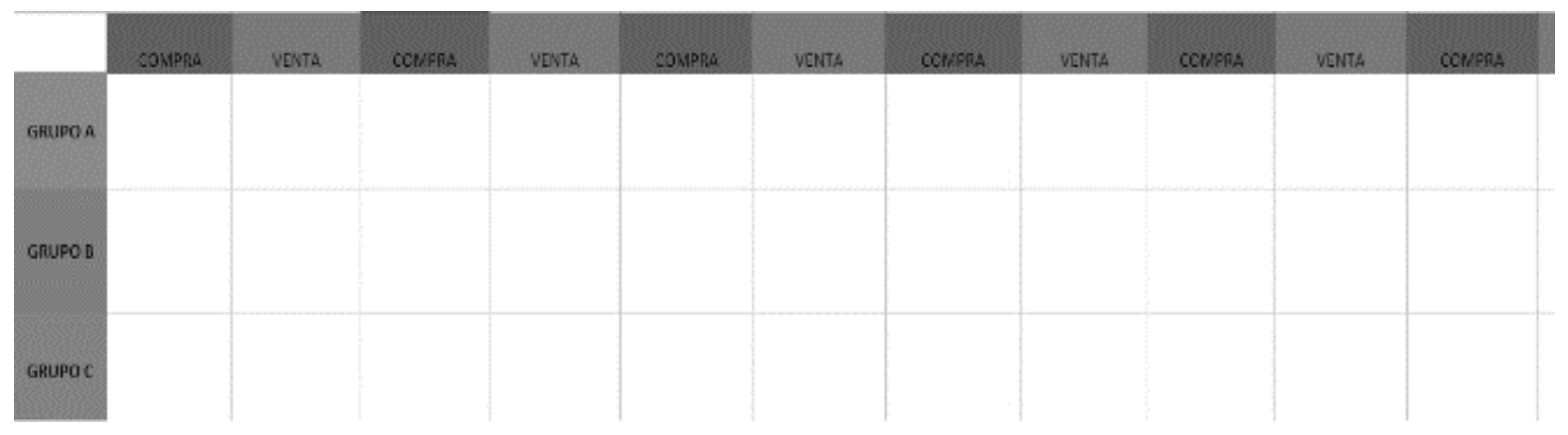

Anexo 2

\section{Sistema de elección y explicación de retos para el desarrollo de la lúdica}

Existen 4 tipos diferentes de retos a realizar, los cuales deben ser explicados a los participantes con el fin de agilizar el desarrollo de la actividad lúdica. Dentro de estas opciones se debe escoger el reto especifico y dar un tiempo de máximo un minuto para su ejecución.

TRIVIA: Este reto consiste en hacer las preguntas de cultura general con 4 opciones de respuesta a los participantes de cada equipo. Estas preguntas poseen una única respuesta correcta, la cual será subrayada dentro de las opciones, con el fin de dar al monitor a cargo una claridad sobre la respuesta correcta.

- ¿Cuál es la flor emblemática de Colombia? A) Brunellia B) Aniba C) Orquídea D) Victoria

- ¿Cuál es el rio más largo del mundo? A) Rio Misisipi B) Rio Nilo C) Rio Amarillo D) Rio Amazonas

- ¿Cuándo se acabó la segunda guerra mundial? A) 1945 B) 1943 C) 1944 D) 1940

- ¿Cómo se llama la capital de Mongolia? A) Ulán Bator B) Wellington C) Helsinki D) Tegucigalpa

- ¿Cuántos huesos tiene un ser humano adulto? A) 210 B) 215 C) 206 D) 204

- ¿En qué lugar del cuerpo se produce la insulina? A) Páncreas B) Hígado C) Estomago D) Pulmones

- ¿Cuál es el color que representa la esperanza? A) Blanco B) Verde C) Morado D) Azul

- ¿A qué país pertenecen los cariocas? A) Argentina B) Bolivia C) Brasil D) Portugal

- ¿Cuál que el primer metal que empleó el humano? A) Estaño B) Oro C) Hierro D) Cobre

- ¿A qué país pertenece la ciudad de Varsovia? A) Polonia B) Noruega C) Canadá D) Nueva Zelanda

- ¿Qué deporte practica profesionalmente Roger Federer? A) Fútbol B) Tenis C) Basquetbol D) Natación

- ¿Cuántos corazones tienen los pulpos? A) 2 B) 3 C) 4 D) 5

- ¿En qué año murió Steve Jobs? A) 2011 B) 2012 C) 2013 D) 2014

- ¿En qué año el ser humano llegó a la luna? A) 1970 B) 1968 C) 1960 D) 1969

- ¿Cuál fue el primer animal en ser clonado? A) Un Perro B) Un Hámster C) Una Oveja D) Un gato

- ¿En qué isla murió Napoleón? A) Isla Margarita B) Isla Santa Elena C) Islas Malvinas D) Islas Galápagos

- ¿Qué es un melómano? A) Un amante de la pintura B) Un amante de los deportes C) Un amante de la música D) Un amante de la poesía

- ¿Quién pintó la noche estrellada? A) Leonardo Da Vinci B) Miguel Ángel C) Yoko Ono D) Vincent van Gogh

- ¿En qué año llegó Cristóbal Colón a América? A) 1492 B) 1940 C) 1540 D) 1350

- ¿Cuál es la cascada más alta del mundo? A) Cascadas del Niagara B) La cascada Kerepakupai Merú C) La cascada Las Tres Hermanas D) La cascada Yumbilla

TARAREAR: Este reto consiste en dar una canción específica a un participante del equipo, este deberá únicamente tararear la canción correspondiente y el equipo deberá adivinar dicha canción. No es válido el uso de otras acciones como mover las manos u hacer otro tipo de gestos.

- Despacito - Luis Fonsi ft. Daddy Yankee

- Con calma - Daddy Yankee, Snow

- Colgando en tus manos - Carlos Baute y Marta Sánchez • Bad Guy - Billie Eilish

- Waka Waka - Shakira

- Callaíta - Bad Bunny, Tainy 
Diego Felipe Lobo-Rueda, Miguel Ángel Lobo-Rueda

El Inversor: un ejercicio gamificado para mercados de valores

- 7 Rings - Ariana Grande

- Shape of you - Ed Sheeran

- Uptown Funk - Mark Ronson feat. Bruno Mars

- Sugar - Maroon 5

- Counting Stars - OneRepublic
- Hello - Adele

- Let Her Go - Passenger

- Dance Monkey - Tones and I

- Thriller - Michael Jackson

- Rolling in the Deep - Adele

DEPATRÁS: Este reto deberá ser desarrollado únicamente por dos participantes del equipo. Se le asignará una palabra o frase la cual deberá ser deletreada en su orden inverso; si alguno de los participantes falla en una letra durante el desarrollo de la actividad, se deberá iniciar desde cero hasta que logren deletrear la palabra o frase o se termine el tiempo estipulado.

- Cohesión

- Púrpura

- Imperturbable

- Antigüedad

- Verídico

- Hidrópico

- Noctámbulos

- Dentífrico

- Bostezar
- Lingüística

- Aurea

- Insensible

- Coherencia

- Omnívoro

- Raquítico

- Alfeñique

- Mezquita

- Extenuación

MÍMICA: Este reto consiste en dar a un participante del equipo una palabra correspondiente a un objeto u actividad, quien deberá hacer que el equipo adivine la palabra asignada usando únicamente expresiones corporales o sonidos. No está permitido que el participante hable durante la actividad o dé pistas al equipo de un método externo al anteriormente mencionado.

- Tiro con arco

- Armar un castillo de arena

- Jugar tenis

- Bailar en una disco

- Tocar guitarra

- Pedir un taxi

- Ganar una carrera

- Inflar globos

- Ser una avioneta

- Tocar la trompeta
- Presentar un examen importante

- Negociar un contrato

- Preparar la comida

- Nadar en una competencia

- Comprar en la tienda

- Pedir un favor 